- Opportunity to familiarise themselves with various accessories in ERCP and EUS

Methods Since 2009 we have organised 9 annual courses which have been attended by $>400$ delegates from all parts of the UK. The programme is a combination of didactic lectures by nurses and endoscopists and breakout sessions with hands on training with models/equipment. The delegates complete a feedback questionnaire at the end of the day. We provide feedback from the last course in 2017.

Results 43 delegates attended the 2017 course from 16 regions in the United Kingdom. 41 (96\%) delegates completed the questionnaire. The average experience of the staff working in endoscopy ranged from 6 months to 22 years. 30/41 $(73 \%)$ of the delegates received funding from their trust. 24/ $41(58 \%)$ had previously attended some form of informal ERCP \pm EUS training. 36/41 (88\%) thought their knowledge was either basic or moderate prior to attending the course but 41/41 (100\%) delegates said that their knowledge had definitely improved after the course. 22/41 (54\%) delegates thought their ability and confidence to assist during an ERCP or EUS was either basic or moderate prior to the course but $38 / 41(93 \%)$ delegates said that it improved after the course. $41 / 41$ (100\%) attendees agreed that all nurses involved in pancreaticobiliary endoscopy should undergo a training course within the first year in post in the endoscopy unit. 100\% of the delegates stated that it gave them an opportunity to interact with colleagues from other units and thereby take back ideas to implement in their respective units.

Conclusions Feedback from the course was positive with evidence of improved knowledge and confidence in assisting in EUS and ERCP. This course has highlighted the advantage of dedicated training in pancreatobiliary endoscopy. NHS trusts appear to be keen to support training nurses and this course goes some of the way to meet the unmet need to provide a comprehensive national training programme for nurses involved in ERCP and EUS.

\section{PTH-139 GASTRIN NOW: ESTABLISHING A TRAINEE LED RESEARCH NETWORK}

Kirsty Nixon*, Katherine White, Wadiamu Gashau, Liam Morris, Rahul Kalla, Simon BorgBartolo. GaTRIN NW (Gastroenterology Trainees Research and Improvement Network North West, Manchester

\subsection{6/gutjnl-2018-BSGAbstracts.538}

Introduction Research, quality improvement and audit are essential parts of effective personal and professional development. Increasing clinical demands and the proposed shortening of training time may limit trainees' involvement in clinical research. Trainee led research and improvement networks (TLRINs) allow us to develop large, cost-effective, multi-site quality improvement and research projects without necessitating time out of programme. They also provide opportunities for tailored input dependent on personal clinical interest.

Aims To assess the feasibility of a new TLRIN, GasTRIN NoW, within Health Education England North West, by conducting a multi-site clinical audit. We aimed to recruit trainee members from $75 \%$ of 16 potential hospital sites, and from these obtain $75 \%$ complete data.
Methods Membership was offered to all gastroenterology specialty trainees in the North West Deanery. All consultants were offered honorary membership. We replicated an audit proforma from the West Midlands TLRIN (GARnet) to assess the management of acute upper gastrointestinal bleeds, collecting prospective data over a one month period. Members were required to sign a constitution to ensure fair acknowledgment of work. Video conferencing enabled regular committee meetings with additional updates to members at regional gastroenterology teaching days.

Results Led by a committee of 6 trainee members, 27 trainees, supported by 25 consultants, from 13 sites (81\%) were recruited to GasTRIN NoW. Complete audit data were received from 10 sites (77\%).

Conclusion The need for and utilisation of TLRINs is increasing in gastroenterology. TLRINs offer trainees the opportunity to experience research and quality improvement in a peer-supported environment alongside clinical work. This will allow them to achieve competencies required for successful attainment of CCT and become more rounded consultants of the future. TLRINs provide a cost effective approach to answer questions of clinical importance and provide innovative change in a coordinated manner. Continuity for data collection and project structure exists even when trainees rotate hospital placements. Our current model has produced encouraging results, building a comprehensive and comparable picture of practice throughout the region with no financial requirements in initial set up.

\section{PTH-140 VIRTUAL REALITY SIMULATION TRAINING IN GASTROINTESTINAL ENDOSCOPY: A COCHRANE SYSTEMATIC REVIEW}

${ }^{1}$ Joanne Plahouras*, ${ }^{2}$ Rishad Khan, ${ }^{3}$ Bradley C Johnston, ${ }^{4}$ Michael A Scaffidi, ${ }^{1,4}$ Samir C Grover, ${ }^{1,5}$ Catharine M Walsh. ${ }^{1}$ University of Toronto, Toronto, Canada ${ }^{2}$ Schulich School of Medicine and Dentistry, Western University, London, Canada ${ }^{3}$ Department of Community Health and Epidemiology, Dalhousie University, Halifax, Canada ${ }^{4}$ Division of Gastroenterology, St. Michael's Hospital, Toronto, Canada ${ }^{5}$ Division of Gastroenterology, SickKids, Toronto, Canada

\subsection{6/gutjnl-2018-BSGAbstracts.539}

Introduction Endoscopy simulation is increasingly being integrated into gastroenterology training programs; however, its effectiveness remains unclear. We sought to determine whether virtual reality (VR) simulation training can complement and/or replace conventional endoscopy training for novices.

Methods Methodological expectations of Cochrane intervention reviews were followed. Health professions, education and computer databases and conference abstracts were searched until July 2017. We included randomised and quasi-randomised clinical trials comparing VR training to conventional training, no training, or alternative simulation methods. Clinical outcomes were considered, including composite score of competency, procedure completion, time, mucosal visualisation, complication rate, discomfort, global rating of competency. We pooled effect size using random-effects meta-analyses.

Results We identified 18 studies with 421 participants. Ten studies compared VR training with no training, 5 with conventional training, 1 with another form of simulation, and 2 compared VR training methods. Table 1 summarises metaanalyses results. Compared with no training, VR training is 